\title{
MANEJO DE COMPLICACIÓN DE TIPO FUGAZZOTTO I PARA ELEVACIÓN DEL PISO DE SENO MAXILAR CON INJERTO INLAY
}

\author{
MANAGEMENT OF COMPLICATION OF TYPE FUGAZZOTTO I FOR THE MAXILLARY \\ SINUS LIFT WITH INLAY BONE GRAFT
}

Juan Domingo Reyes Doimi ${ }^{1 a}$, Timothy Crosby Bertorini ${ }^{1 b}$, Miguel Coz Fano ${ }^{1 \mathrm{c}}$

\begin{abstract}
RESUMEN
Las rupturas producidas en el momento de la manipulación de la membrana de Schneider cuando se realizan elevaciones de piso de seno maxilar son muy comunes en nuestra práctica, por lo cual es sugerida una nueva alternativa de tratamiento para las perforaciones, en este caso la de tipo I de Fugazzoto, producida al momento de colocar un injerto en bloque tipo Inlay. Esta técnica es predecible y óptima para la solución de imprevistos presentados durante la cirugía.
\end{abstract}

Palabras clave: Elevación de piso de seno maxilar; Membrana de Schneider; Complicaciones quirúrgicas, Implantes dentales.

\section{ABSTRACT}

Perforations produced during the manipulation of the Schneiderian membrane on sinus lift procedures are common in our practice. That is the reason why a new alternative is suggested in this article for sinus lift membrane perforations. On this case, a Fugazzotto type I perforation happened at the moment of the placement of an inlay block bone xenograft. This technique is predictable and optimal for fixing membrane ruptures during surgery.

Keyword: Maxillary sinus lift; Schneiderian membrane; Surgical complications; Dental implant

\footnotetext{
${ }^{1}$ Universidad de San Martin de Porres. Maestría en periodoncia.

${ }^{a}$ Magister. Especialista en periodoncia. Docente de Post-grado.

${ }^{B}$ Docente de Post-grado. Especialidad en Rehabilitación Oral.

${ }^{\mathrm{C}}$ Magister. Especialista en periodoncia. Docente coordinador.

Correspondencia:

Este es un artículo Open Access distribuido bajo la licencia Creative Commons Atribución-NoComercialCompartirlgual 4.0 


\section{INTRODUCCIÓN}

La colocación de implantes dentales en el área distal de la maxila edéntula es frecuentemente un problema. Debido a la reabsorción significativa luego de las extracciones dentarias ${ }^{(1)}$ y la neumati- zación del seno maxilar, muchas veces no se encuentra suficiente volumen óseo para asegurar la estabilidad primaria necesaria de los implantes (2-4). Elevar el seno maxilar logra incrementar la altura ósea como lo demostraron Boyne \& James ${ }^{(5)}$ y Tatum ${ }^{(6)}$ utilizando injertos óseo autólogo.

La eficacia en tratamientos con elevaciones de piso de seno maxilar incrementando la disponibilidad ósea en la región maxilar posterior ha sido bien documentada por medio de evaluaciones histológicas animales y humanas como reportes de caso clínicos ${ }^{(7,8)}$. Las tasas de éxito para colocar implantes en estas áreas aumentadas son comparables con aquellas utilizando implantes en áreas no regeneradas, tanto en regiones posteriores de la maxila como en otras áreas de la boca.

Nunes et al. ${ }^{(9)}$ en un análisis de tipo tomográfico determinaron que el promedio del grosor de la membrana de Schneider en el ser humano es de $1.50 \mathrm{~mm}$

Sin embargo, la presencia de perforaciones a nivel de la membrana de Schneider en el momento de realizar la cirugía, frecuentemente inducen a que el operador replantee la posibilidad de elevar el piso del seno maxilar con colocación simultánea de implantes durante la misma visita.

Vlassis \& Fugazzotto (10,11) propusieron una clasificación para reparar perforaciones de la membrana sinusal,la cual fue simplificada a tres tipos de ruptura (I, II y III) en el año 2003 en base a la experiencia personal clínica adquirida por los autores, en un esfuerzo por facilitar la manipulación de esta ocurrencia y lograr un efecto predecible. La clase I se encuentra a cualquier nivel la región apical de la ventana pre-parada. La clase II en cualquier parte lateral de la ventana y la clase III en cualquier área comprendida dentro de los límites de la preparación.

En el siguiente caso clínico, veremos una propuesta de manejo en una perforación de Fugazzotto tipo I, así como los resultados del seguimiento de 5 años.

\section{REPORTE DE CASO}

\section{Selección del Paciente}

Paciente de sexo femenino de 62 años, edéntula parcial sin presentar signos de enfermedad periodontal (fig. 1), que acude en Agosto del año 2011 a la Clínica Especializada en Odontología de la Universidad de San Martín de Porres debido a su incomodidad para la masticación. La paciente presentaba un buen estado general. No manifestó antecedentes históricos generales de consideración. Radiográficamente se detectó una marcada neumatización del seno maxilar del lado derecho, así como ausencia de las piezas 4.5, 4.6, 4.7 y 4.8., y un reborde óseo remanente de $2 \mathrm{~mm}$. de espesor (fig. 2), por lo cual se le indicó realizar un procedimiento de elevación del piso de seno maxilar con la técnica de ventana lateral ${ }^{(6)}$, para posteriormente colocar dos implantes dentales y así devolverle la función perdida. La paciente firmó un consentimiento informado aceptando las condiciones del tratamiento.

La paciente recibió 2 gr. de Amoxicilina una hora previa a la cirugía. La cirugía se realizó bajo anestesia local (lidocaína con epinefrina 1:100,000). Con una hoja de bisturí número $15 \mathrm{C}$ se hizo una incisión de tipo supracrestal con dos incisiones liberantes para facilitar la visibilidad del campo operatorio y se decoló un colgajo a espesor total. Para preparar la ventana se utilizó una fresa redonda diamantada de $3 \mathrm{~mm}$ de diámetro, marca Karl Schumacher® para determinar el perímetro de la ventana hasta evidenciar la membrana de Schneider y poder empezar el proceso de levantamiento del piso de la cavidad, para lo cual se utilizaron las curetas número 1,2 y 3 del sistema SLA de Neobiotech®. (Figs. 3 y 4). Luego de determinar el tamaño del nuevo piso del seno maxilar, se acondicionó un bloque de hueso de bovino de composición mixta (Orthogen, Baumer®) (fig. 5), el cual luego de adaptarse seccionándolo, fue fijado por medio de un tornillo desde el aspecto oclusal (parafuso Conexâo® de $1.5 \mathrm{~mm} \times 10 \mathrm{~mm}$ ) (fig. 6). La manipulación excesiva del campo operatorio llevó a una ruptura de la porción superior de la membrana de Schneider (Clase I de Fugazzotto), por lo cual se optó por desplazar el extremo inferior de la misma en sentido apical y fijarlo con una sutura reabsorbible de ácido poliglicólico en la porción ósea por medio de una perforación en la parte superior de la ventana (Fig. 7). Una vez fijada la membrana, se colocó $1 \mathrm{gr}$. de hueso bovino mixto particulado de granulación gruesa (Orthogen, Baumer®) con la finalidad de ocupar el resto de la cavidad preparada. Se colocó por encima una membrana Genderm reabsorbible 


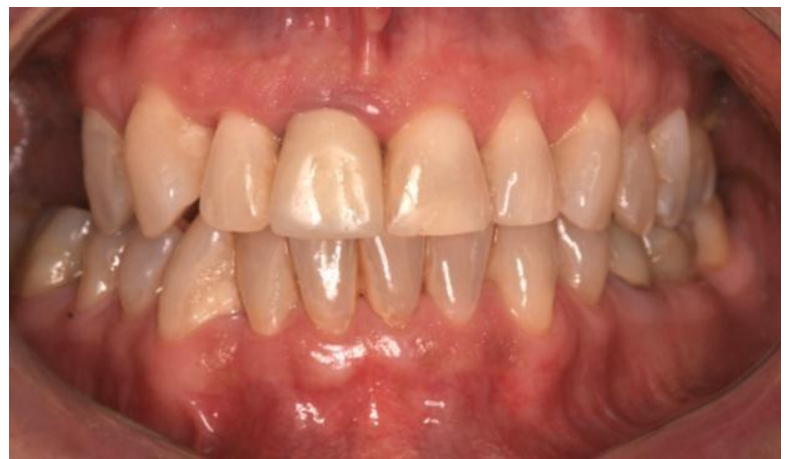

Figura 1. Paciente edéntula parcial sin signos de enfermedad periodontal

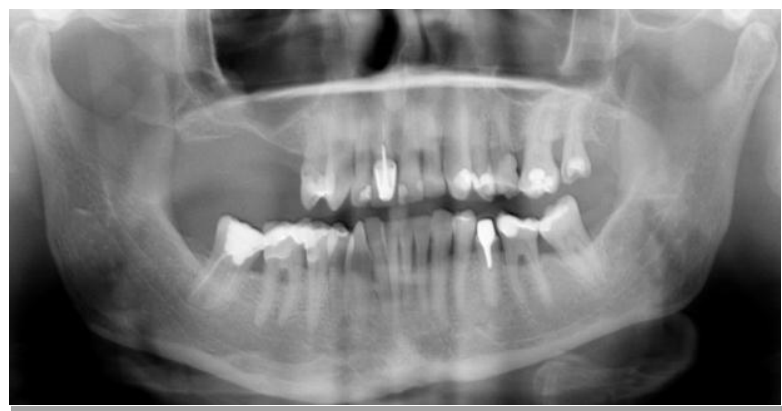

Figura 2. Neumatización del seno maxilar a nivel del cuadrante I

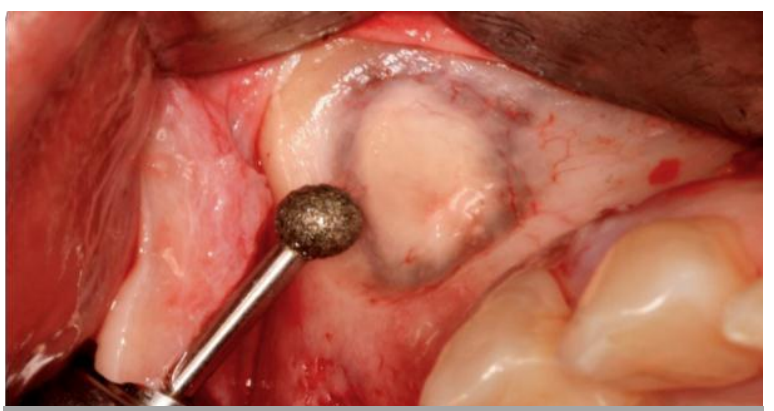

Figura 3. Preparación de la ventana con una fresa diamantada de $3 \mathrm{~mm}$

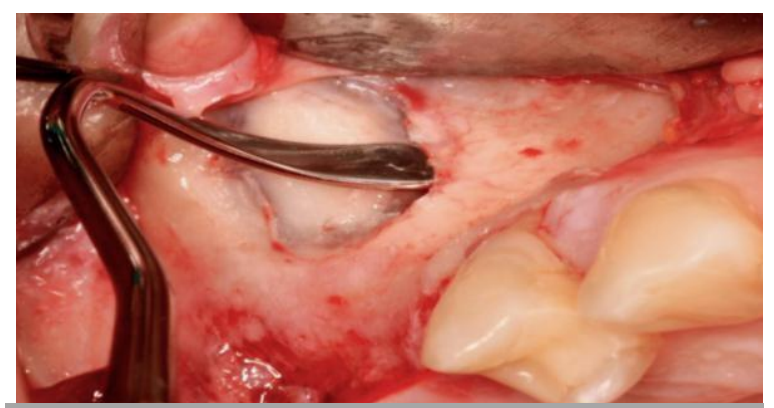

Figura 4. Desprendimiento de la membrana de Schneider

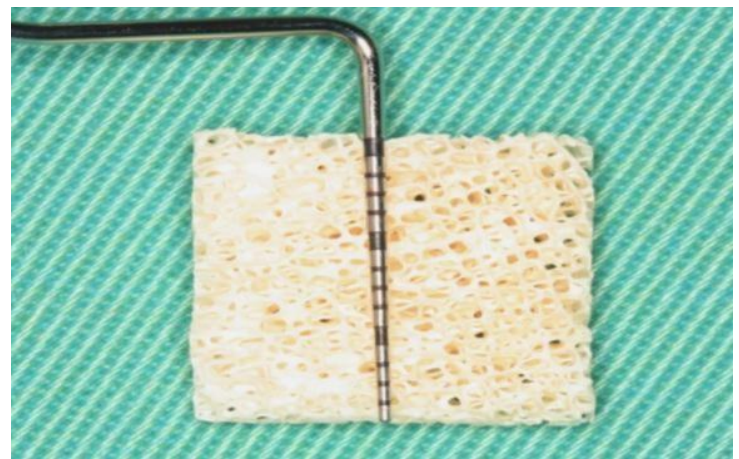

Figura 5. Bloque de Xenoinjerto listo para ser preparado y acondicionado.

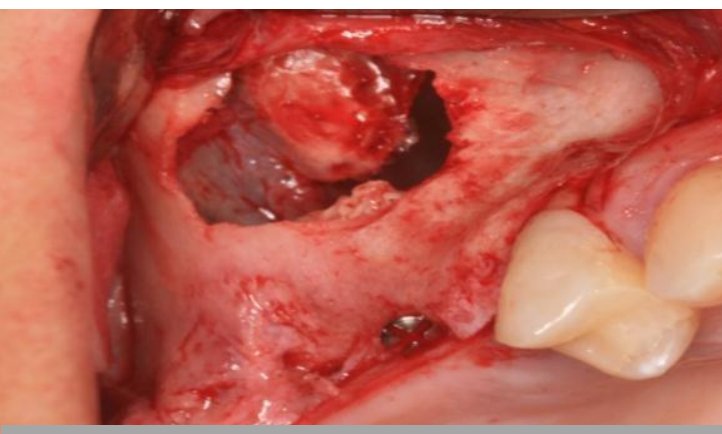

Figura 6. Fijación del bloque con un tornillo desde el aspecto oclusal y ruptura evidente

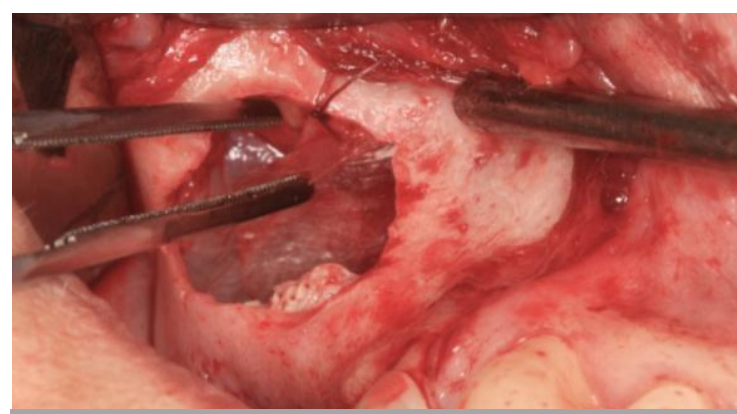

Figura 7. Sutura del borde inferior de la ruptura a la cortical superior

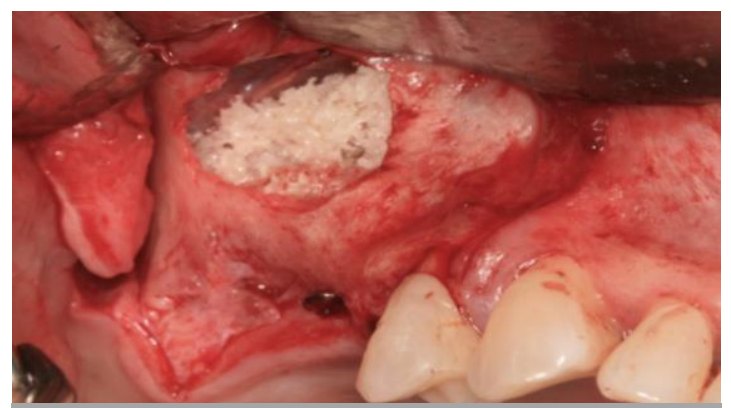

Figura 8. Colocación de xenoinjerto particulado en la cavidad sinusal. 
de colágeno Baumer® y se cerró el colgajo por primera intención después de dividirse. Inmediatamente después se tomó una radiografía panorámica en donde se evidenció la presencia del biomaterial así como el tornillo de fijación (Figuras.8 y 9).
La paciente no volvió a sus controles sino hasta septiembre del año 2014, en donde se pudo retirar el tornillo de fijación y colocar dos implantes de diámetro regular con una longitud de $11.5 \mathrm{~mm}$. tipo Master Porous (Conexâo®) previo control tomográfico ( Figs. 10 y 11).
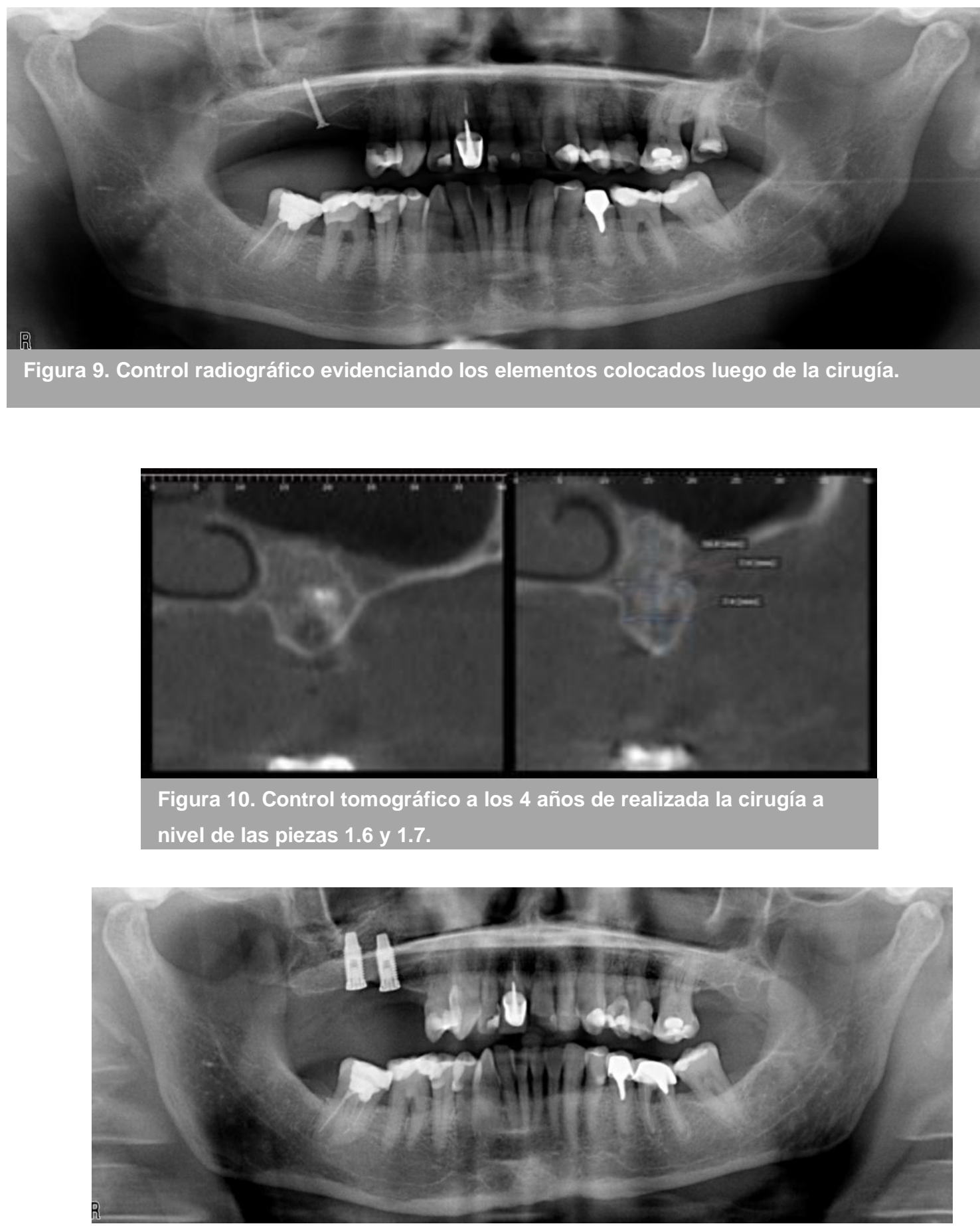

Figura 11. Implantes colocados a nivel de piezas 1.6 y 1.7 . 


\section{Protocolo protésico}

En mayo del año 2015 la paciente acudió para la segunda fase quirúrgica de los implantes. Se tomó la impresión con la técnica de cubeta abierta sobre la plataforma de los implantes. En la misma fecha se procedió a tomar el modelo del antagonista, un registro de mordida y arco facial para el montaje de los modelos.

Se colocaron los análogos de los implantes y se realizó el vaceado con falsa encía y yeso extra duro. Una vez articulados los modelos, se seleccionaron los pilares, tipo COC ( Pilar Preparo ) con un hombro de $2 \mathrm{~mm}$. Los pilares coc son una opción viable para la rehabilitación sobre im-plantes según Stona (12), quien explica la resistencia a las fracturas de las coronas sobre este tipo de pilares prepararon los pilares para lograr paralelismo y se redujeron en altura para dar el espacio oclusal de las coronas.

Los modelos ya listos, fueron escaneados con el sistema Cad/Cam de Sirona Cerec. En el software se vieron las dimensiones de los modelos y se delimitó el hombro del pilar para darle el perfil de emergencia de las coronas lo más estético posible El programa brindó una anatomía preliminar y manualmente se modificó para que cumpla los parámetros anatómicos y oclusales deseados.
Terminado el proceso de diseño se mandó a tallar el bloque seleccionado de Emax (disilicato de litio) Lt de color A3, de la marca ivoclar vivadent. Según Nawafleh ${ }^{(13)}$, el disilicato de litio es un material que puede soportar la fatiga oclusal de una mordida convencional. Una vez tallado el puente se pulió el vástago y se le aplicó el glase para colocarlo en el horno para su cristalización y glaseado. El día de la instalación, se retiraron los cicatrizales y se colocaron los pilares preparo, se controló radiográficamente y verificado el asentamiento se procedió a torquear los pilares con $20 \mathrm{~N} / \mathrm{Cm}$.

La prueba del puente se realizó inicialmente sin ocluir, solo para verificar el asentamiento sobre los hombros. Tras verificar la pasividad se controló la oclusión y movimientos excéntricos.

Satisfechos con la adaptación del puente, se trató la superficie interna del puente con ácido fluor-hidrico y silano, la rehabilitación se fijó definitivamente con un cemento resinoso (rely $x$ u200 de la $3 M$ ESPE).(Figura 12) Se realizó un control radiográfico al finalizar la rehabilitación del caso (Figura 13).

El control se realizó al mes de la instalación y no se observaron irregularidades en la rehabilitación por lo que se dio de alta a la paciente.
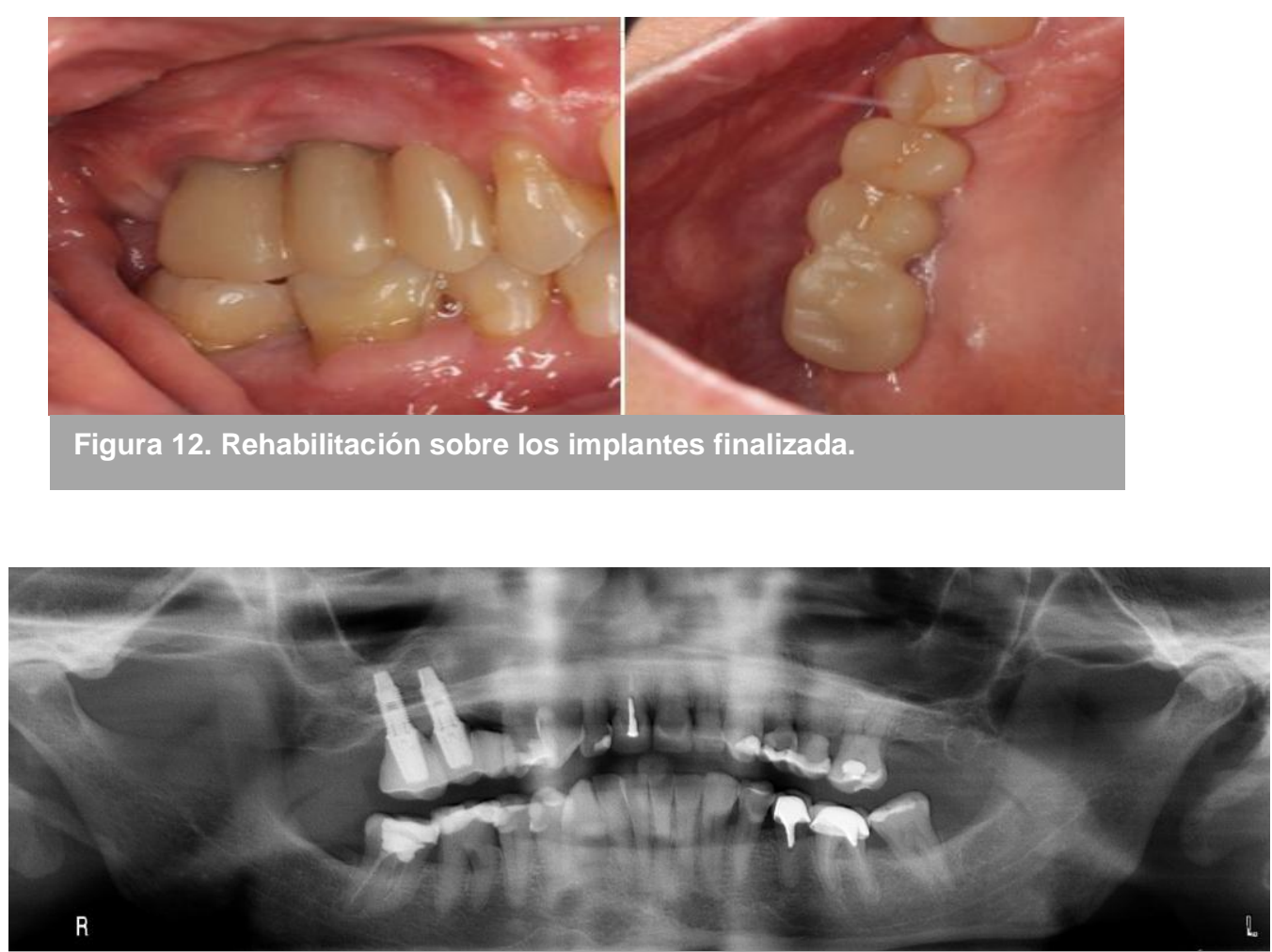

Figura 13. Control radiográfico al finalizar la rehabilitación del caso. 


\section{DISCUSIÓN}

Mientras el debate continua acerca de la importancia de la presencia o ausencia de rupturas en la membrana de Schneider afectando en el proceso de regeneración del hueso injertado en el seno maxilar, no hay duda que una membrana de Schneider intacta brinda un área adecuada para la recepción de un biomaterial a ser colocado junto con un buen coágulo sanguíneo. Sin embargo, la presencia de una ruptura de la membrana durante el procedimiento no debe ser vista como una contraindicación para continuar con el procedimiento planeado. Sin duda el mejor tratamiento de las perforaciones es prevenirlas. El diagnostico preoperatorio, plan de tratamiento, cuidados a la hora de preparar la ventana, técnica con el instrumental adecuado y ejecución mínimamente traumática, reducirán las posibilidades que esto ocurra, sin embargo aún con todos los cuidados, pueden ocurrir. La técnica descrita para el manejo de las rupturas de Fugazzotto tipo I pretende brindar una opción válida y simple para la comodidad del operador y así contar con una alterna-tiva simple, viable, de buena predictibilidad y bajo costo.

\section{CONCLUSIONES}

El presente trabajo confirma que la ruptura de la membrana de Schneider de tipo Fugazzotto I, suturada al borde superior de la ventana puede ser considerada como una alternativa exitosa si se siguen los protocolos adecuados. Las ventajas de este procedimiento en una sola fase ofrecen las condiciones adecuadas para poder finalizar el tratamiento originalmente planeado sin tener que modificar ni retardar la planificación. La colocación de implantes bajo un reborde con la membrana de Schneider perforada y suturada no implicó diferencia alguna. Se recomienda realizar ensayos clínicos e histológicos que demuestren las ventajas de aplicar esta nueva opción como alternativa de tratamiento.

Contribuciones de autoría: JDR, TCB y MCF participaron en el diagnóstico y tratamiento del paciente, redactaron y aprobaron la versión final del artículo.

Fuente de financiamiento: autofinanciado.

Conflictos de interés: Los autores declaran no tener conflictos de interés en la publicación de este reporte de caso.

\section{REFERENCIAS BIBLIOGRÁFICAS}

1. Tallgren, A. The continuing reduction of the residual alveolar ridges in complete denture wearers: a mixedlongitudinal study covering 25 years. Journal of Prosthetic Dentistry. 1972; 27: 120-132.

2. Branemark P. I., Adell, R. \& Albrektsson, T. An experimental and clinical study of osseointegrated implants penetrating the na- sal cavity and maxillary sinus. Journal of Oral and Maxillofacial Surgery. 1984; 42: 497-505.

3. Listrom, R. D. \& Symington, J. M. Osseo integrated dental implants in conjunction with bone grafts. Journal of Oral and Maxillofacial Surgery. 1988; 17: 116-118.

4. Blomqvist JE1, Alberius $P$, Isaksson $S$, Linde $A$, Obrant K. Importance of bone graft quality for implant integration after maxillary sinus reconstruction. Oral Surg Oral Med Oral Pathol Oral Radiol Endod. 1998 Sep;86(3):268-74.

5. Boyne PJ, James RA. Grafting of the maxillary sinus floor with autologous marrow and bone. J Oral Surg.1980 Aug;38(8):613-6.

6. Tatum $\mathrm{H}$ Jr. Maxillary and sinus implant reconstructions. Dent Clin North Am. 1986 Apr;30(2):207-29.

7. Smiler DG, Holmes RE. Sinus lift procedure using porous hydroxyapatite: A preliminary clinical report. $\mathrm{J}$ Oral Implantol. 1987; 13: 239-253.

8. Olson JW, Dent CD, Morris HF, Ochi S. Long-term assessment (5-71 months) of endosseus dental implants placed in the augmented maxillary sinus. Ann Periodontol. 2000; 5: 152-156.

9. Nunes LS, Bornstein MM, Sendi P, Buser D. Anatomical Characteristics and dimensions of edentulous sites in the posterior maxillae of patients referred for implant therapy. Int J Periodontics Restorative Dent. 2013 MayJun; 33(3): 337-45.

10. Vlassis J, Fugazzotto PA. A classification system for sinus membrane perforations during augmentation procedures with options for repair. J Periodontol 1999; 70: 692-699.

11. Fugazotto PA, Vlassis J. A simplified classification and repair system for sinus membrane perforations. J Periodontol. 2003; 74: 1534-1541.

12. Stona D, Burnett LH Jr, Mota EG, Spohr AM. Fracture resistance of computer-aided design and computeraided manufacturing ceramic crowns cemented on solid abutments. J Am Dent Assoc. 2015 Jul; 146(7): 501-7.

13. Nawafleh N1, Hatamleh M2, Elshiyab S1, Mack F1. Lithium Disilicate Restorations Fatigue Testing Parameters: A Systematic Review. J Prosthodont. 2016 Feb; 25(2): 116-26. 